\title{
Article défini et métaphore : étude descriptive
}

† Naoyo FURUKAWA

\section{Note de l'éditeur}

Monsieur Naoyo Furukawa (1942-2018), qui a quitté ce monde trop tôt et trop brutalement, nous a laissé deux articles qui n'avaient pas vu le jour avant son décès. L'un sur l'adjectivité sera publié dans la revue Travaux de linguistique. C'est l'autre, qui ne connaissait pas encore de projet de publication, que nous voudrions reproduire ci-dessous. Nous remercions sincèrement Madame Naomi Taniguchi, fille de monsieur Furukawa, de nous avoir confié le manuscrit.

(Jun-ya Watanabe)

\section{Introduction}

Le point de départ de cette étude se trouve dans l'observation de l'emploi de l'article défini dans des syntagmes nominaux tels qu'on les trouve dans les deux types d'exemples suivants :

(1) Ne lui retournez pas le couteau dans la plaie, Octavie. Donnez-moi plutôt un paquet de tabac comme d'habitude, et deux petits verres de rhum pour trinquer avec madame Toriotte, ça la requinquera. (G. Chepfer, Saynètes, paysanneries, Frantext)

(2) Car si ce roi Teuton n'était pas châtié, /Tout ce que l'homme appelle espoir, progrès, pitié, /Fraternité, fuirait de la terre sans joie ; /Car César est le tigre et le peuple est la proie,/ [...].

(V. Hugo, L'Année terrible, Frantext)

L'hypothèse de départ est simple : dans la mesure où le référent du SN le couteau et celui du $\mathrm{SN}$ le tigre échappent, respectivement, à l'extension des couteaux et à celle des tigres au sens propre, l'emploi de l'article défini dans ces SN se justifie par la seule mise en jeu de l'intension de chaque nom; en bref, il s'agit d'un emploi intensionnel.

L'environnement syntaxico-sémantique n'est pas le même dans les deux SN. Le SN le couteau fait partie de l'expression (ou locution) retourner le couteau dans la plaie, alors que ce n'est pas le cas pour le SN le tigre. La différence entre (1) et (2) peut aussi être décrite en termes de métaphorisant/métaphorisé en disant que dans (2) le métaphorisant le tigre a pour métaphorisé César, ce qu'exprime d'ailleurs, de manière explicite, la construction attributive, tandis que l'expression retourner le couteau dans la plaie dans (1) est moins facile à saisir en ces termes.

L'objet premier de cette étude étant d'observer l'emploi intensionnel, emploi essentiel, sinon exclusif, de l'article défini dans les SN métaphoriques, nous examinerons, en premier lieu, l'emploi de l'article défini dans quelques expressions. En dépassant le niveau de l'article défini, nous étudierons, en second lieu, la construction du type César est le tigre (et le peuple est la proie), qui recourt à un contexte contrastif pour 
son occurrence. En troisième lieu, nous considérerons le type exemplifié par «le lion [...]. Tarquin, le roi superbe, est le lion», qui fait appel à une stratégie de reprise. Nous verrons que le contraste et la reprise contribuent à compléter la faible référentialité (ou force référentielle) d'un SN défini métaphorique en position d'attribut et à rendre celui-ci référentiellement équivalent au SN sujet. Nous étudierons, en quatrième lieu, le type représenté par Le requin est le tigre de la mer, type qui ne requiert ni le recours au contraste ni le recours à la reprise, et qui peut être accepté tel quel hors contexte. Enfin, nous montrerons que généralement, la construction à attribut défini métaphorique constitue une phrase d'identification et, plus particulièrement, celle d'identification illusoire ${ }^{1}$, et qu'en revanche, la construction à attribut indéfini métaphorique, illustré par César est un tigre, est structurellement sous-déterminée à cet égard.

\section{L'article défini dans les expressions (ou locutions)}

On commencera par considérer l'expression retourner le couteau dans la plaie. Le choix de cette expression parmi d'autres n'est pas fortuit, car il s'agit d'une expression qui présente une transparence sémantique malgré son statut d'expression classée en tant que telle : «faire souffrir (qqn) en évoquant ce qui lui est très pénible, en ranimant une douleur, etc. $»^{2}$,et dans laquelle chaque élément constitutif de l'ensemble n'est pas complètement figé, ce qui permettra donc de mieux observer l'emploi de l'article défini que dans le cas du figement total ${ }^{3}$. Le verbe est soit retourner, soit remuer, soit enfoncer, soit planter. Le déterminant précédant le nom complément d'objet direct est soit $l e$, soit un. Le nom complément d'objet direct est soit couteau, soit poignard. Le régime de la préposition dans est soit la plaie, soit le cour, modifié ou non.

Une expression est une séquence exprimant un sens qui ne correspond pas à l'addition de sens de chaque élément constitutif. Dans le cas de l'expression en cause, néanmoins, son opacité sémantique est peu marquée, voire inexistante, ce qui se manifeste par son degré de figement peu élevét. Nous sommes donc ici en présence d'un cas limite entre expressions et séquences libres. Le statut d'expression de cette séquence se justifie par le fait que celle-ci sert à rendre un sens métaphorique (ou figuré), non pas le sens littéral (ou propre), et qu'elle est une séquence stéréotypée pour décrire une action ou une situation déterminée. Dans ce qui suit, nous allons examiner son aspect de non-expression (ou séquence libre), ce qui permettra de mieux observer l'élément constitutif qu'est le SN complément d'objet direct.

\subsection{Le SN le couteau dans l'expression retourner le couteau dans la plaie}

Voyons à nouveau l'exemple (1). Il ne serait pas pertinent d'isoler ici l'élément le couteau de l'ensemble et de s'interroger sur ce à quoi il réfère métaphoriquement, dans la mesure où la phrase veut

\footnotetext{
${ }^{1}$ L'expression «identification illusoire» est empruntée à Tamba (1999: 229), qui l'utilise quand elle parle de la construction du type cette fille est une tortue (comparée à celle du type cette fille est comme une tortue).

2 A. Rey et S. Chantreau (2003) : Dictionnaire des expressions et locutions, Collection Les Usuels, Le Robert.

${ }^{3}$ Cf. Lamiroy et Klein (2005). Le titre de cet article («Le problème central du figement est le semi-figement») représente bel et bien l'essentiel du problème du figement. Sur le semi-figement, cf. le numéro 53 de LINX, 2005.

${ }^{4}$ Sur l'opacité sémantique, cf. G. Gross (1996).
} 
dire globalement quelque chose comme : Ne la tourmentez pas, elle souffre déjà assez $z^{5}$, ce qui s'explique certes par la propriété caractéristique des expressions en général. Mais, dans l'exemple suivant :

(3) Pour Marie-Alice, dont la vie était tout entière concentrée sur son enfant, chaque heure maintenant retournait le couteau dans la plaie. (P. Bourget, Cruelle énigme, Frantext)

il ne serait pas déraisonnable de mettre en relation l'élément le couteau avec le sujet chaque heure et de remarquer la valeur métaphorique de celui-là. On peut en effet considérer qu'il s'agit là d'une relation métaphorisant/métaphorisé. L'expression peut aller jusqu'à se désintégrer partiellement, en perdant le verbe retourner (ou remuer, etc.), et approcher de la construction attributive métaphorique, celle illustrée par (2) (César est le tigre et le peuple est la proie), comme en témoigne l'exemple ci-dessous :

(4) J'ai subi cette cure d'abord pendant un an, avec des fortunes diverses. Ce fut, au moins durant les premiers temps, le couteau dans la plaie. (M. Leiris, L'Age d'homme..., Frantext)

Interrogeons-nous maintenant sur la valeur de l'article défini dans l'expression en cause. On remarquera que l'article défini ici n'est pas un «déterminant figé » ${ }^{6}$, car il peut céder sa place à un article indéfini, comme dans :

(5) Madame... madame, pardonnez-moi... j'ai été folle... car je viens de retourner un couteau dans la plaie de votre cœur... et Turquoise, la voix entrecoupée, les yeux pleins de larmes, se jeta aux genoux de Baccarat. (P.-A. Ponson du Terrail, Rocambole, Frantext)

L'alternance des articles défini et indéfini s'observe aussi dans l'expression jeter lelun voile sur, comme l'attestent les exemples ci-dessous :

(6a) Il ne faut pas que l'on pose de cloison entre ma «littérature» et ma «politique», que l'on m'enferme dans Venise ou dans ma Lorraine, que l'on jette le voile sur mon boulangisme. Je ne me prête pas à ce morcellement. (M. Barrès, Mes Cahiers, Frantext)

(6b) Voilà bien la pensée de Marx et de Engels, à cette date. Je sais que l'on cherche maintenant à jeter un voile sur la brutalité de ces textes. (J. Jaurès, Études socialistes, Frantext)

Que signifie l'alternance des deux articles qui n'affecte pas fondamentalement le sens de l'expression? Elle ne va pas sans rappeler la situation sémantique dite générique, telle qu'on la trouve par exemple dans L'homme/Un homme est faillible.

\subsection{Le $N$ métaphorique et $l e N$ générique : intension sélective et intension intégrale}

Dans nos études antérieures, nous avons mis en avant que cet usage dit générique de l'article défini (ex. : L'homme est faillible) pouvait être assimilé à son emploi intensionnel ${ }^{7}$. Notre hypothèse ici est que cet emploi intensionnel s'applique aussi à l'expression en cause, dans la mesure où l'usage métaphorique

\footnotetext{
${ }^{5}$ Pour Simatos (1997 : 83), il s'agit d'un «prédicat lexicalisé structuralement complexe » et d'« une structure dans laquelle le GN objet n'a pas de référence actuelle, n'est pas un argument, et corrélativement, dans laquelle le verbe ne figure pas comme prédicat. ».

${ }^{6}$ Cf. M. Gross $(1985,1988,1993)$.

${ }^{7}$ Et ceci non seulement dans sa forme singulière mais aussi, un peu paradoxalement, dans sa forme plurielle. Pour le détail, cf. Furukawa (1997).
} 
d'un nom nous fait quitter le monde extensionnel de celui-ci (ou l'ensemble constitué d'éléments que l'on désigne par ce nom), met donc en jeu l'intension seule.

Il existe néanmoins une différence entre le cas de l'usage métaphorique et celui de l'usage générique. Dans ce dernier cas, l'intension qu'il implique est totale ou intégrale, puisqu'elle constitue la définition même d'un genre ou d'une espèce. Ainsi, l'article défini le dans la phrase :

(7) Le chat est carnivore.

« renvoie à l'intension de chat, c'est-à-dire à l'ensemble des propriétés qui font qu'un chat est un chat. » ${ }^{8,9}$. Si l'on définit l'intension d'un nom comme l'ensemble des propriétés qui font qu'un $\mathrm{N}_{i}$ est un $\mathrm{N}_{i}$, l'intension requise dans le premier cas est, en revanche, partielle ou sélective. Cette intension sélective correspond à ce que le locuteur considère comme caractéristique d'un nom en usage métaphorique. Ainsi, dans l'intension du nom couteau, lequel se définit comme «instrument tranchant servant à couper, composé d'une lame et d'un manche » (Petit Robert), n'entre en jeu que la propriété «lame» en tant que partie qui peut provoquer une douleur aiguë ${ }^{10}$.

Cela dit, toutefois, la mise en jeu de l'intension sélective dont on a parlé ci-dessus doit être attribuée au nom, et non pas à l'article défini. Au niveau de ce dernier, il n'existe pas de différence entre l'usage métaphorique et l'usage générique. Ainsi, le SN le tigre dans (2) (César est le tigre et le peuple est la proie) gagne à être confronté au même SN dans la construction (8) ci-dessous :

(8) César est au peuple ce que le tigre est à la proie.

construction qui exprime le même contenu sémantique ou, du moins, la même relation sémantique qu'en (2). On remarquera que le tigre en position de sujet est un SN généralement appelé générique et que les deux usages de l'article défini, celui dans le tigre de (2) et celui dans le tigre de (8), se rejoignent ainsi en ce qu'ils sont intensionnels.

Enfin, l'examen du SN le couteau dans l'expression retourner le couteau dans la plaie nous a permis de constater que l'article défini le est utilisé dans son emploi intensionnel. Dans ce qui suit, nous étudierons, en dépassant le niveau de l'article défini, la construction à attribut défini métaphorique, et nous nous interrogerons sur le sens de l'existence de contraintes (ou conditions) qui s'imposent à l'occurrence de le $N$ métaphorique en position d'attribut.

\footnotetext{
${ }^{8}$ Martin (1986: 190). Sur la conception restrictive de l'emploi intensionnel de l'article défini, cf. Leeman (2004: 33-35) : tests syntaxiques à l'appui, elle ne reconnaît l'emploi en cause que dans des locutions telles que prendre l'eau, en excluant le cas de l'usage générique (par ex. L'eau dans L'eau est indispensable à la vie).

${ }^{9}$ Le prédicat carnivore en (7), qui contribue certes à la définition même du sujet le chat, n'entame pourtant pas l'intégralité potentielle de l'intension du nom d'espèce chat.

${ }^{10}$ Sur «l'usage sélectif » de la métaphore, Martin (1983 : 197) écrit à juste titre : «Dire de l'homme qu'il est un loup, c'est sélectionner dans les prédications propres au loup celle qui convient au prédicat homme et éliminer transitoirement les autres. [...] En disant que Sophie a une démarche de lionne, je ne veux d'aucune façon faire accroire que Sophie marche à quatre pattes !».
} 


\section{La construction avec $L E N$ attribut et ses modes d'occurrence}

Nous faisons remarquer que la séparation de la séquence César est le tigre d'avec le contexte (à droite) en (2) n'est pas sans incidence sur son acceptabilité. Cela ressort clairement surtout quand on la compare à son correspondant indéfini :

(9a) ?César est le tigre.

(9b) César est un tigre.

La phrase (9a) ne se laisse pas facilement accepter, alors que (9b) s'interprète sans problème. Cela permet de considérer que le SN le tigre ne peut produire à lui seul une interprétation intensionnelle et que pour cela même, il demande un renfort approprié.

\subsection{Le contraste}

Il faut d'abord observer que dans (2) la mise en jeu de l'intension du SN le tigre est assurée par le contraste avec la proie. Il s'agit ainsi de l'idée de férocité ou de voracité ou encore de force. La mise en jeu de la seule intension par la stratégie du contraste s'observe également dans :

(10) Entre le projet en plâtre et la statue exécutée en marbre, on pouvait, disait Claude Vignon, défigurer un chef-d'œuvre ou faire une grande chose d'une mauvaise. Le plâtre est le manuscrit, le marbre est le livre. (H. de Balzac, La Cousine Bette, Frantext)

La relation sémantique contenue dans la séquence en italique de (10) peut être exprimée, comme en (2), par :

(10’) Le plâtre est au marbre ce que le manuscrit est au livre.

\subsection{Le $N$ intensionnel et sa familiarité avec même et par excellence}

Notons ici que le nom abstrait, que l'on peut considérer comme essentiellement intensionnel, s'accorde bien avec même, comme l'illustre (11):

(11) Mlle Victorine avait de l'esprit et réfléchissait beaucoup, elle était la fraîcheur même. (Stendhal,

Vie de H. Brulard, Frantext)

La séquence la fraîcheur même exprime « une qualité possédée au plus haut point » (Petit Robert). On peut considérer que ce sens provient de ce que la fonction de même dans le $N$ même consiste à marquer qu'il s'agit exactement de l'être ou de la chose en question. Ainsi, cette fonction s'observe également auprès de noms qui ne sont pas des noms abstraits, comme l'illustre (12) :

(12) Aubryet lui envoie ses feuilletons, à ce qu'elle me dit. Je ne savais pas qu'elle sût lire dans l'imprimé ! Bonne fille, après tout, et qui l'est restée. Vraie fille, bien bête et qui veut être prise au sérieux : c'est la fille même. (E. et J. Goncourt, Journal, Frantext)

Il s'agit d'une image des filles, à laquelle correspond exactement la fille en question.

Par ailleurs, l'expression par excellence contribue particulièrement à consolider l'interprétation intensionnelle de le $N$, comme en témoigne l'exemple qui suit : 
(13) Nous avons plus ou moins oublié le sens original du mot évangile. Il signifie : la bonne nouvelle. Exactement comme la bible était le livre par excellence, l'évangile apportait aux hommes la bonne nouvelle par excellence. (E. Gilson, L'Esprit de la philosophie médiévale, Frantext)

La séquence le livre par excellence occupe la position d'attribut, position qui se prête à la lecture intensionnelle. Ce qui n'empêche toutefois pas que le $N$ par excellence figure en position référentielle. Dans l'exemple ci-dessous :

(14) La rubrique par excellence, c'est la chronique, le plus souvent quotidienne, que les journalistes et même le public ne désignent que par le nom de son auteur. (Anonyme, La civilisation écrite, Frantext)

la rubrique par excellence occupe la position d'élément initial détaché, position thématique par excellence et donc référentielle. Les deux faits ne sont nullement contradictoires. Car, si l'on admet que le $N$ réfère toujours à quelque chose, le livre par excellence en (13) et la rubrique par excellence en (14) réfèrent à l'intension de chaque nom. Dans le même ordre d'idées, on peut dire que les phrases la bible était le livre par excellence en (13) et La rubrique par excellence, c'est la chronique, [...] en (14) sont des phrases d'identification dans la mesure où chaque sujet (ou thème) et son attribut sont référentiels.

\subsection{Le $N$ métaphorique et son accord ou désaccord avec même et par excellence}

Constatons tout d'abord que les deux phrases suivantes sont également acceptables :

(15a) César est un tigre.

(15b) César est un héros.

Le remplacement de un par le entraîne cependant une différence de degré d'acceptabilité (ou d'inacceptabilité) entre les deux :

(16a) ?César est le tigre.

(16b) (?)César est le héros.

La phrase (16b) est certes moins bonne que (15b), mais meilleure que (16a). L'ajout après le nom de même ou par excellence, matériaux qui contribuent, rappelons-le, à renforcer la lecture intensionnelle, rend la phrase (16b) parfaite :

(17a) César est le héros même.

(17a') César est le héros par excellence.

D'autre part, il ne parvient pas à sauver (16a). Les avis divergent sur l'acceptabilité de (17b-b') :

(17b) (?)César est le tigre même.

(17b’) (?)César est le tigre par excellence.

La réticence qu'éprouvent certains locuteurs à accepter (17b-b') s'expliquerait par l'intension sélective qui caractérise les SN métaphoriques. En effet, logiquement, César ne peut satisfaire à l'intension intégrale de le tigre même, puisqu'il est un être humain. Il ne peut non plus prétendre au statut de le tigre par excellence au détriment de vrais tigres. 
La réticence qui vient d'être analysée pourrait aussi s'expliquer par une raison sémantico-pragmatique. Imaginons une échelle de degré de ressemblance où le plus haut degré se confond avec l'identité même. On peut considérer que l'énonciation des phrases (17b-b') implique que dans cette échelle soit dépassé le degré représenté par César est un tigre, étant donné que le plus haut degré de ressemblance y est atteint. Ainsi, la réticence que l'on peut éprouver face à (17b-b') s'expliquerait en supposant que la proposition véhiculée par César est un tigre, qui n'a rien d'évident, n'est pas de nature à être impliquée mais constitue celle qui vaut la peine d'être assertée ${ }^{11}$.

Cette situation est similaire, sinon identique, à celle que présente le contraste le N/ce $N$ dans la reprise immédiate. Corblin (1983 : 123) signale, en donnant la paire d'exemples suivante :

(18a) Un arbre dressait ses branches tordues non loin de là. Il décida de passer la nuit près $d u$ compagnon.

(18b) Un arbre dressait ses branches tordues non loin de là. Il décida de passer la nuit près de ce compagnon.

que «l'anaphore définie est impossible, alors que l'anaphore démonstrative est possible». Ici, on a bien affaire à une métaphore. L'inacceptabilité de (18a) s'explique par le coup de force qui consiste à présupposer arbre = compagnon : présupposition qui n'a rien d'évident. Nous faisons remarquer que la forme acceptable ce compagnon constitue, à la différence de le compagnon, une assertion, et non une présupposition. Il faut en effet considérer que ce $N$ est la forme nominale abrégée de la structure classificatoire du type «c'est un $\mathrm{N}$ » et qu'en l'occurrence, ce compagnon s'analyse comme la forme abrégée de la phrase assertive : «c'est un compagnon $»^{12}$.

En revenant aux phrases (17b-b'), nous faisons remarquer que la suppression du coup de force présuppositionnel les rend, sinon parfaites, du moins meilleures :

(19a) César est un tigre... c'est le tigre même.

(19b) César est un tigre... c'est le tigre par excellence.

\subsection{Le type «le lion [...]. Tarquin, le roi superbe, est le lion » : cas de reprise}

Dans les sections précédentes, nous avons vu que l'occurrence de le $N$ métaphorique en position d'attribut n'était pas sans contrainte et qu'elle était rendue possible par la stratégie du contraste ; ce qui nous a conduit à considérer que le contraste met en valeur l'intension de $N$ et qu'ainsi, l'emploi de l'article le est intensionnel et ce, indépendamment du fait que l'intension mise en jeu n'est pas intégrale mais sélective.

Or, le $N$ métaphorique peut apparaître en position d'attribut sans l'aide du contraste. Voyons l'exemple ci-dessous :

\footnotetext{
${ }^{11}$ Ce n'est pas le cas pour les phrases du type elle est la fraîcheur même. La bonne formation de ces phrases tient au fait qu'il ne s'agit pas de noms métaphoriques mais de noms essentiellement qualificatifs.

${ }^{12}$ Cette analyse s'inspire de Kleiber (1984). Cf. aussi Furukawa (1990).
} 
(20) On dit que le lion, qui s'abreuve de sang, quand il trouve en chemin un cadavre gisant, après avoir flairé, d'une avide narine, s'il ne reste plus d'âme au fond de la poitrine, repousse avec dédain le corps inanimé, et, réservant pour mieux son courroux affamé, cherche ailleurs une proie, [...]. Tarquin, le roi superbe, est le lion; de sorte qu'étant lui le lion, je suis la bête morte, et que Tarquin-lion, quand il m'eut bien tourné, ne trouvant nulle part une âme, a pardonné. (F. Ponsard, Lucrèce, Frantext)

On a bien affaire à un cas de reprise, mais de nature un peu particulière. En effet, il n'est pas pertinent de parler ici de coréférence. Il conviendrait plutôt de parler de reprise intensionnelle ou, si l'on préfère, lexicale, ou encore conceptuelle. Ce qu'il est essentiel de remarquer, c'est que l'occurrence du défini le lion dans Tarquin, le roi superbe, est le lion ne pourrait être justifiée sans cette reprise.

Il en est de même dans l'exemple (21) :

(21) Avez-vous jamais vu les courses d'Angleterre ? On prend quatre coureurs, quatre chevaux sellés ; on leur montre un clocher, puis on leur dit : allez! Il s'agit d'arriver, n'importe la manière. L'un choisit un ravin, l'autre un chemin battu. Celui-ci gagnera, s'il ne rencontre un fleuve ; celui-là fera mieux, s'il n'a le cou rompu. Tel est l'amour, Silvio ; l'amour est une épreuve; il faut aller au but, la femme est le clocher. (A. de Musset, À quoi rêvent les jeunes filles, Frantext)

La différence avec le précédent consiste simplement en ce que le défini le clocher ne met pas en jeu la qualité intrinsèque d'un clocher mais le rôle qui est situationnellement attribué à celui-ci, ce que montre la difficulté de la substitution de l'indéfini un clocher au défini le clocher dans la femme est le clocher. Il faut aussi remarquer que c'est la reprise qui permet l'occurrence du défini le clocher dans la femme est le clocher.

Le $N$ métaphorique en position d'attribut va de pair, on l'a vu, avec le contraste, d'une part et d'autre part, avec la reprise. Cela semble signifier qu'il est, tel quel, référentiellement faible ou instable. On peut considérer que le rôle du contraste et celui de la reprise consistent tous deux à renforcer ou à compléter la faible référentialité (ou force référentielle) d'un SN défini métaphorique en position d'attribut et à rendre ce SN attribut référentiellement équivalent au SN sujet, ce qui veut dire que la construction à attribut défini métaphorique constitue une phrase d'identification et, plus particulièrement, une phrase d'identification illusoire $^{13}$.

\section{Le type Le requin est le tigre de la mer : interprétation attributive}

Le type que nous allons étudier ici est celui qui ne requiert ni le recours au contraste ni le recours à la reprise et qui peut être accepté tel quel hors contexte. C'est la forme $l e N$ de $S N$ comme dans :

(22) Le requin est le tigre de la mer. J'en ai vu de plus de dix pieds de longueur. (B. de Saint-Pierre,

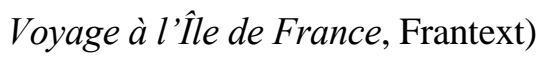

${ }^{13}$ Sur la force référentielle, cf. Kleiber (1981: 114 et ss) et Riegel (1985 : 56 et ss). 
La question de savoir si l'emploi de l'article défini est intensionnel ou non n'est plus pertinente, puisqu'il ne s'agit pas de le $N$ tout court et que l'on a affaire à un emploi dit cataphorique. La distinction qui semble pertinente ici est celle entre l'usage attributif et l'usage référentiel, donc celle au niveau du syntagme nominal, et non du déterminant ${ }^{14}$. Nous verrons dans ce qui suit que l'interprétation qui s'impose dans le type en question est celle attributive (ou prédicative), et non référentielle.

L'exemple (22) est à rapprocher de l'exemple suivant, où le nom-tête est un nom propre :

(23) M. de Chateaubriand était le Napoléon de la littérature. (A. de Lamartine, Nouvelles confidences, Frantext $)^{15}$

On peut supposer que le mécanisme à l'œuvre en (22) est fondamentalement le même que celui en (23). Il convient ici de citer l'étude de Gary-Prieur (1994) et celle de Jonasson (1994) sur le nom propre. Elles donnent des exemples tels que :

(24a) Le Tino Rossi du rock (il s'agit de Richard Antony) (Gary-Prieur : 117)

(24b) Le Boris Vian de la S. F. américaine... [= Robert Scheckley] (Jonasson : 216)

Gary-Prieur (1994 : 118) écrit :

L'interprétation de ces expressions fonctionne sur le mode de l'analogie: Richard Antony, par exemple, [...], est, dans le domaine du rock, l'analogue de Tino Rossi [...] dans le domaine de la chanson. ».

Et elle cite Jonasson (1991, repris dans 1994) :

Pour le type le Boris Vian de la S. F. américaine, la métaphorisation du Npr consisterait donc en un rapprochement entre deux domaines encyclopédiques distincts. À l'intérieur d'un de ces domaines le référent original du Npr occupe une position privilégiée, qui l'a rendu plus ou moins célèbre. Lorsque cette structure ou figuration est appliquée à l'un des domaines encyclopédiques dans lesquels s'inscrit le référent topique, il s'avère que la position de celui-ci dans ce nouveau domaine peut être comparée à celle du référent original dans le domaine initial. (p. 118).

Il est à noter que sont en jeu, dans chaque cas, deux domaines distincts, «domaine initial » et «nouveau domaine ». Voyons l'exemple suivant, exemple modifié de (24a) :

(24a') Richard Antony est le Tino Rossi du rock.

On remarquera que la phrase est composée de trois termes : Richard Antony, Tino Rossi et le rock, et non de quatre termes, puisque le domaine initial, qui est celui de la chanson, est implicite. Si l'on explicite le domaine initial, la relation sémantique entre les quatre termes pourra être exprimée par :

(24a") Richard Antony est au rock ce que Tino Rossi est à la chanson.

L'application de ce type de paraphrase à (23) permet d'exprimer la relation sémantique qu'il contient par:

(23’) Chateaubriand est à la littérature ce que Napoléon est à la politique (ou au domaine militaire)

14 Cf. Donnellan (1966).

15 L'exemple est de Keiichi Naganuma. 
Il y a tout lieu de penser que cette analyse est applicable au cas du nom commun. La relation sémantique contenue dans (22) peut être exprimée comme suit :

$\left(22^{\prime}\right)$ Le requin est à la mer (= monde des animaux marins) ce que le tigre est à la terre (= monde des animaux terrestres).

Quelle est alors la différence, si différence il y a, entre le cas du nom commun et celui du nom propre ? Elle consisterait en celle de degré de saillance entre les deux, dans la mesure où le référent original du nom propre occupe une position privilégiée et donc saillante dans un domaine par rapport auquel il est évalué, tandis que dans le cas du nom commun, son référent original n'évoque pas nécessairement le domaine auquel il appartient, à cause de son degré de saillance moins élevé.

Certes, le nom-tête tigre dans le tigre de la mer de (22) possède une saillance suffisamment élevée pour lui permettre d'occuper la position thématique qu'est la position de sujet dans la paraphrase (22'). Cependant, dans :

(25) Les champignons, c'était la chair même de la forêt, une chair dont la saveur tenait de l'arbre et de la terre. (A. de Chateaubriant, Monsieur des Lourdines, Frantext)

il semble difficile de concevoir une paraphrase de la forme : le champignon est à la forêt ce que la chair est à $x$, parce qu'il y a difficulté à déterminer $x$, c'est-à-dire, le domaine auquel se rapporterait la chair, à la différence du champignon, qui appartient lui au domaine forestier. Il ne s'agit donc pas ici de la relation entre la position du champignon dans la forêt et celle qu'occuperait la chair par rapport à un domaine quelconque, mais plus simplement et plus directement, de la ressemblance formelle ou gustative (épaisseur, mollesse, etc.) entre le champignon et la chair.

Cela nous incite à supposer que la séquence chair même de la forêt en (25) constitue une séquence plus ou moins serrée et, en quelque sorte, une intension composée ou une intension discursivement construite (chair-de-la-forêt), grâce au degré de saillance moins élevé du nom-tête chair. Mais ce qu'il faut dire, c'est que l'idée d'intension composée est susceptible de s'appliquer également aux autres exemples cités, et que la différence se réduit à celle de degré de saillance du nom-tête entre eux. Ainsi, dans :

(26a) le Napoléon de la littérature

(26b) le tigre de la mer

(26c) la chair de la forêt

on pourrait dire que le degré de saillance du nom-tête décroît dans l'ordre $\mathrm{a}>\mathrm{b}>\mathrm{c}$. Il faut enfin souligner que (26a-c) ont ceci de commun qu'ils s'interprètent de manière attributive.

\section{Le type César est un tigre : phrase d'identification ou non ?}

La structure sémantique de la séquence César est le tigre (et le peuple est la proie) pourrait être représentée comme le schéma (27), dans lequel le signe d'égalité exprime le verbe être :

(27) César = le tigre

Il s'agit donc de l'identification référentielle. 
On peut alors se demander ce qu'il en est de la phrase César est un tigre. Disons d'emblée qu'elle est structurellement sous-déterminée à cet égard, dans la mesure où le $\mathrm{SN}$ attribut un tigre est non référentiel et où, par conséquent, il est susceptible de former avec le verbe être une unité prédicative comparable à celle constituée par être tyrannique. Nous considérons que c'est cette sous-détermination structurelle qui permet de parler soit de «métaphores d'appartenance ou d'inclusion » (Kleiber, 1983 : 104), soit d' « équivalence » (Martin, 1983 : 190), soit d'«identification» (Martin, 1983 : 191; Tamba, 1999 : 235). En donnant la formule le N1 est un N2 (+ expansion), Tamba (1999: 231) fait remarquer à juste titre qu'avec l'adjonction de l'expansion à un N2, on passe de la classification à l'équivalence, comme le montrent les énoncés (28a-b) ci-dessous, qui sont d'ailleurs des énoncés non métaphoriques :

(28a) Le martin-pêcheur est un oiseau. (Tamba, 1999 : 228)

(28b) Le martin-pêcheur est un bel oiseau aux couleurs métalliques. (ibid. : 232)

Elle écrit :

Alors qu'en (25) [= (28a)], par exemple, la relation lexicale d'hyperonymie sert à classer les martins-pêcheurs dans la catégorie plus générale des oiseaux, selon la formule analytique : le N1 est un $N 2$, en (25') [= (28b)], oiseau ne fonctionne plus comme une unité lexicale isolée, correspondant à un degré taxinomique. Mais il constitue la tête nominale d'une unité sémantique complexe : « un-bel-oiseau-aux-couleurs-métalliques », posée comme l'explicitation du sens attribué au N1. Une telle équivalence ressort de la réversibilité de ces formulations, contrairement à ce qui se passe avec les classifications, par définition asymétriques.(p. 232-233).

Et elle donne la phrase suivante :

(28c) Un bel oiseau aux couleurs métalliques est un martin-pêcheur. (ibid. : 233)

Il ne s'agit pas ici de la vraie permutation du sujet et de l'attribut, puisque l'attribut en (28c) est un martin-pêcheur, et non le martin-pêcheur. Mais avec l'emploi du pronom neutre ce, l'attribut le martin-pêcheur devient possible :

(28c') Un bel oiseau aux couleurs métalliques, c'est le martin-pêcheur.

L'essentiel est de remarquer que la séquence un bel oiseau aux couleurs métalliques occupe la position de sujet en (28c) et celle d'élément initial détaché en (28c') et qu'elle est donc pleinement référentielle.

Cette analyse s'applique également au cas de le N1 est un N2 (+ expansion), où N2 est un nom métaphorique. Voyons l'exemple qui suit :

(29) Qui dirait, mademoiselle, que cet homme, qui a l'air d'une jeune fille, est un tigre à griffes d'acier qui vous déchire une réputation comme il doit déchirer vos peignoirs quand vous tardez à les ôter. (H. de Balzac, Les Illusions perdues, Frantext)

La séquence en italique se rapproche indéfiniment de la phrase d'identification, dans la mesure où la quasi-permutation du sujet et de l'attribut avec l'emploi du pronom neutre $c e$ est possible : 
(29') un tigre à griffes d'acier qui vous déchire une réputation comme il doit déchirer vos peignoirs quand vous tardez à les ôter, c'est cet homme

\section{Conclusion}

Cette étude a commencé par l'observation de l'article défini dans des expressions comme retourner le couteau dans la plaie. L'hypothèse de départ était que l'emploi de l'article défini dans le SN le couteau se justifie par la seule mise en jeu de l'intension du nom couteau, en bref, il s'agit là d'un emploi intensionnel, dans la mesure où le référent du SN en cause échappe à l'extension des couteaux au sens propre. Le rapprochement de cet usage avec l'usage dit générique (ex. : L'homme est faillible) nous a permis de constater que ces deux usages se rejoignent en ce qu'ils sont intensionnels. Nous avons vu que la différence entre le $N$ métaphorique et le $N$ dit générique consiste en ce que le premier met en jeu l'intension partielle ou sélective du nom, tandis que le second implique l'intension totale, et que néanmoins, l'usage de l'article défini lui-même reste le même, la différence entre les deux se réduisant au niveau du nom, et non au niveau de l'article.

En dépassant le niveau de l'article défini, nous avons étudié la construction du type César est le tigre (et le peuple est la proie), qui recourt à un contexte contrastif pour son occurrence. On peut dire qu'il s'agit ici aussi d'un emploi intensionnel et que le contraste mis en œuvre constitue une stratégie qui contribue à compléter la faible référentialité d'un SN défini intensionnel et à rendre celui-ci référentiellement équivalent au SN sujet, ce que demande par ailleurs la construction à attribut défini elle-même. Nous avons ensuite examiné le type illustré par «le lion [...]. Tarquin, le roi superbe, est le lion», dans lequel l'occurrence du SN attribut le lion est rendue possible par le fait que celui-ci est la reprise de la première occurrence de le lion et qu'il se trouve ainsi référentiellement étoffé et donc suffisamment fort pour équivaloir au SN sujet. On peut dire que la reprise est une autre façon de remplir l'exigence de la construction qui veut que la référentialité de le $N$ attribut soit équivalente à celle du SN sujet. Étant donné qu'il s'agit ici d'un cas de reprise, on n'a plus affaire à l'emploi intensionnel proprement dit. À la différence des deux types précédents, le type représenté par Le requin est le tigre de la mer ne requiert ni le recours au contraste ni le recours à la reprise, et peut être accepté tel quel hors contexte, ce qui signifie que le SN le tigre de la mer est référentiellement en quelque sorte autosuffisant, autrement dit, sa référentialité est suffisamment forte pour équivaloir à celle du SN sujet. De toutes ces analyses, il ressort que la construction à attribut métaphorique de forme le $N$ (+ expansion) constitue une phrase d'identification et plus particulièrement, une phrase d'identification illusoire. Enfin, il a été avancé que la construction du type César est un tigre est structurellement sous-déterminée quant au fait de savoir s'il s'agit là d'une phrase d'identification ou non. 


\section{Références bibliographiques}

Corblin, F. (1983), « Défini et démonstratif dans la reprise immédiate », Le français moderne, 51, 2 : 118-134.

Donnellan, K. (1966), « Reference and definite descriptions », Philosophical Review, 75 : 281-304.

Furukawa, N. (1990), " Cet animal construit des barrages: le SN générique et ses modes de référence», L'information grammaticale, $47:$ 3-10.

Furukawa, N. (1997), «Les Glaneuses de Millet: emploi intensionnel de LE(S)», Revue de sémantique et pragmatique, $2: 169-181$.

Gary-Prieur, M.-N. (1994), Grammaire du nom propre, Paris, Presses Universitaires de France.

Gross, G. (1996), Les expressions figées en français, noms composés et autres locutions, Paris, Ophrys.

Gross, M. (1985), « Sur les déterminants dans les expressions figées », Langages, 79 : 89-117.

Gross, M. (1988), «Les limites de la phrase figée », Langages, 90 : 7-22.

Gross, M. (1993), «Les phrases figées en français », L'Information grammaticale, 59 : 36-41.

Jonasson, K. (1994), Le nom propre, constructions et interprétations, Louvain-la-Neuve, Duculot.

Kleiber, G. (1981), Problèmes de référence : descriptions définies et noms propres, Paris, Klincksieck.

Kleiber, G. (1983), « Métaphores et vérité », LINX, 9 : 89-130.

Kleiber, G. (1984), «Sur la sémantique des descriptions démonstratives ». Linguisticae Investigationes, VIII, 1 : 63-85.

Kleiber, G. (1999), «Une métaphore qui ronronne n'est pas toujours un chat heureux », N. Charbonnel \& G. Kleiber, éds., La métaphore entre philosophie et rhétorique, Paris, Presses Universitaires de France : 83-134.

Lamiroy, B. \& J. R. Klein (2005), « Le problème central du figement est le semi-figement », LINX, 53 : 135-154.

Leeman, D. (2004), Les déterminants du nom en français, Paris, Presses Universitaires de France.

Martin, R. (1983), Pour une logique du sens, Paris, Presses Universitaires de France.

Martin, R. (1986), «Les usages génériques de l'article et la pluralité », J. David \& G. Kleiber, éds., Déterminants: syntaxe et sémantique, Paris, Klincksieck : 187-202.

Riegel, M. (1985), L'adjectif attribut, Paris, Presses Universitaires de France.

Simatos, I. (1997), « Référence et argumentalité du GN dans les locutions verbales », P. Fiala et al., éds., La locution : entre lexique, syntaxe et pragmatique, Paris, Publications de l'INALF, Klincksieck : 77-102.

Tamba-Mecz, I. (1981), Le sens figuré, vers une théorie de l'énonciation figurative, Paris, Presses Universitaires de France.

Tamba, I. (1994), «Une clé pour différencier deux types d'interprétation figurée, métaphorique et métonymique », Langue française, $101: 26-34$.

Tamba, I. (1999), «La femme est-elle une fleur comme le bleuet est une fleur? Métaphore et classification : les structures en «le N1 est un N2 » , N. Charbonnel \& G. Kleiber, éds., La métaphore entre philosophie et rhétorique, Paris, Presses Universitaires de France : 207-235.

(Professeur émérite de l'Université de Tsukuba) 